Journal of Computer Science 7 (12): 1921-1927, 2011

ISSN 1549-3636

(C) 2011 Science Publications

\title{
The Role of Electrical Pulse Shapes in Electrical and Optical Chromatic Dispersion Compensation
}

\author{
Mohammad Syuhaimi Ab-Rahman and Majid Moghaddasi \\ Department of Electrical Electronics and Systems, \\ Faculty of Engineering and Built Environment, \\ Universiti Kebangsaan Malaysia, \\ 43600 UKM Bangi, Selangor, Malaysia
}

\begin{abstract}
Problem statement: The performance of the optical network is highly dependent to Chromatic Dispersion (CD). CD has been a big issue when higher bitrate or longer distance is desired. Many methods have been represented to mitigate or eliminate this phenomenon. A wide range of optical and electrical compensation has been investigated. Approach: In this study we evaluate the efficiency of the electrical pulse shapes including (NRZ,RZ and Triangular form) in electrical compensation with Feed Forward-Decision Feedback Equalizer (FFE-DFE) technique and optical compensation; Dispersion Compensation Fiber. Results: It was compared the mentioned situation with Quality factor versus increasing the distance with different pulse shapes. It was observed that in Electrical compensation there is no considerable with different pulse shapes while in optical compensation NRZ is definitely more susceptible than others. Optical and electrical compensators were compared regarding to jitter and optical compensator showed much better performance. Conclusion: chromatic compensation was implemented with different pulse shapes and RZ pulse shape with optical compensation showed best performance.
\end{abstract}

Key words: Chromatic dispersion, optical compensation, electrical compensation, pulse shape, DCF, NRZ versus RZ, quality factor, eye diagram, Inter Symbol Interference (ISI)

\section{INTRODUCTION}

Optical Network is a rather new technology which provides the best performance for high speed and long haul communication systems. Especially with appearance of new technologies such as Voice over IP (VoIP) and IPTV and needing for higher data rate and longer distance is more desired. Typical reach of optical networks in metropolitan area networks is around $300 \mathrm{~km}$. The network capacity can simply be increased by increasing the data rate from the existing 2.5-10 $\mathrm{Gb} \mathrm{sec}^{-1}$ (Bhandare et al., 2010). But there is some impairment that challenges getting this desire. Impairment such as attenuation, dispersion and nonlinear effects are main factors that make decrement in performance of the optical networks. After invention of EDFA, attenuation is not a big issue anymore and finding solutions for vanishing or at least mitigation of dispersion decreased as much as possible. and nonlinear effects is more studied.

Chromatic dispersion: Fiber impairments such as chromatic dispersion (CD) severely impact the performance of high speed optical fiber transmission systems (Xu et al., 2009). It is one of the most important parameters and should be carefully analyzed during their design. In order to avoid power penalty due to nonlinear effects, in a long-hall transmission system optical fibers with small but nonzero chromatic dispersion is required. $\mathrm{CD}$ is a major issue in single mode fibers that causes Inter Symbol Interference (ISI) in the pulse. It occurs because the spectral components of the emitted light traverse with different velocities. It is called Group Velocity Dispersion. For calculating the pulse broadening because of group velocity dispersion, we consider following assumptions: $\mathrm{L}$ is the length of a single mode fiber; $\omega$ is the frequency of a particular component which reach at the end of fiber after a time delay Eq. 1:

$$
\mathrm{T}=\mathrm{L} / \mathrm{V}_{\mathrm{g}}
$$

While Vg is group velocity that defined as Eq. 2:

$$
V_{g}=(d \beta / d \omega)^{-1}
$$

Corresponding Author: Mohammad Syuhaimi Ab-Rahman, Department of Electrical, Electronics and Systems, Faculty of Engineering and Built Environment, University Kebangsaan Malaysia, 
If we consider $\beta=\check{\mathrm{n}} \times \omega / \mathrm{c}$ and we know $\mathrm{V}_{\mathrm{g}}=\mathrm{c} /$ $\check{\mathrm{n}}_{\mathrm{g}}$, so we have Eq. 3:

$\check{\mathrm{n}}_{\mathrm{g}}=\check{\mathrm{n}}+\omega(\mathrm{d} \check{\mathrm{n}} / \mathrm{d} \omega)$

Here n̆g is named group index. Because the group velocity is dependent to the frequency, pulse broadening will be eventuated. It happens simply because different spectral components of the pulse do not arrive simultaneously at the fiber output. Now if $\Delta \omega$ is spectral with of the pulse, the extension of the pulse broadening is represented by Eq. 4 :

$\Delta \mathrm{T}=(\mathrm{dT} / \mathrm{d} \omega) \Delta \omega=\mathrm{L}\left(\mathrm{d}^{2} \beta / \mathrm{d} \omega^{2}\right)$

$\Delta \omega=\mathrm{L} \beta_{2} \Delta \omega$

While we define:

$\beta_{2}=d^{2} \beta / d \omega^{2}$

As group velocity parameter. It determines how much a pulse will be broadened inside a fiber.

If we want to calculate it based on wavelength we earn Eq. 5:

$\Delta \mathrm{T}=\mathrm{D} \mathrm{L} \Delta \lambda$

While:

$\mathrm{D}=-2\left(\pi \mathrm{c} / \lambda^{2}\right) \beta_{2}$

$\mathrm{D}$ is dispersion parameter and the unit of that are $\mathrm{ps} / \mathrm{nm}-\mathrm{km}$.

There are two factors which contribute chromatic dispersion; material dispersion and waveguide dispersion. It means we can write GVD based on these two phenomena. If we represent Material Dispersion parameter with DM and waveguide dispersion with DW (Agrawal, 2002) we will have Eq. 6:

$\mathrm{D}=\mathrm{D}_{\mathrm{M}}+\mathrm{D}_{\mathrm{W}}$.

Self phase modulation: It is one kind of non-linear effect that is created because light encounters with different refractive index when traverses along the fiber due to Kerr effect. Consequently phase shift will be produced and it eventuate a change of pulse frequency spectrum Refractive Index of many optical material has little dependency to the optical Intensity Eq. 7:

$\mathrm{n}=\mathrm{n}_{0}+\mathrm{n}_{2} \mathrm{I}=\mathrm{n}_{0}+\mathrm{n}_{2}\left(\mathrm{P} / \mathrm{A}_{\mathrm{eff}}\right)$

Here $\mathrm{n}_{0}$ is our ordinary refractive index, $\mathrm{n}_{2}$ is named nonlinear index coefficient. The factor $\mathrm{n}_{2}$ is variable in silica from $2.2-3.4 \times 10-8 \mu \mathrm{m}^{-2} \mathrm{~W}$. Here $\mathrm{n}$ is real refractive index regarding to SPM effect. SPM converts optical power fluctuation to phase fluctuation in same wave (Keiser, 2003).
Chromatic dispersion compensation methods: For elimination or mitigating chromatic dispersion, several techniques have been represented. We can classify them into two main groups: (1) Optical compensation technique; that is premier technique for that goal. (2) Electrical compensation; that was presented as auxiliary for compensation, but with improving the technology, it has been a considerable technique.

Electrical compensator: It is an attractive method to compensate dispersion at electrical part of receiver (or transmitter). It is a simple technique that doesn't need any changes in optical transmitting or receiving and also doesn't have considerable loss. Any network changes or adding new devices in the network can be done easily because of adaptive capability of electrical compensator. But there are some disadvantages of this system, for example circuits have limitation in speed compare to optical ones. There are several techniques for Electrical equalizer, such as: Feed Forward Equalizer (FFE), Feed Forward-Decision Feed Back Equalizer (FFE-DFE), Non Linear Feed ForwardDecision Feedback Equalizer (NL-FFE-DFE) and Maximum Likelihood Sequence Estimator (MLSE).

Optical compensator: It is premier dispersion compensation method. Also optical compensation comprises several techniques; such as DCF, Fiber Brag Grating (FBG), Mid-span phase Conjugation. Since in our experiment, we implement optical compensation with DCF, so we look explain about it.

DCF is a conventional with high performance solution to mitigate CD. SSMF has low positive dispersion, while DCF has high negative one. In other words dispersion coefficient is much higher than ordinary fiber with opposite sign (usually between -80 , $-100 \mathrm{ps} \mathrm{km}^{-1} \mathrm{~nm}$ ). Commonly DCF length is used 4-20 times shorter than the transmission fiber length, so overall the arithmetic summation of the dispersion in the whole fiber will be zero. But on the other hand its attenuation is higher than ordinary fiber $(0.5-0.6 \mathrm{~dB}$ $\mathrm{km}^{-1}$ ). So we need to amplify the signal. The efficiency of using DCF in CD compensation in presence of SPM was shown by Nuyts et al. (1997). DCF can be put at the beginning of main fiber, this method is named precompensation, or after fiber, which is named postcompensation and also subsequently at end and beginning of several fibers, which is named mixcompensation. It was shown that mix-compensation and then post-compensation have better performance than pre-compensation (Hu et al., 2010). 


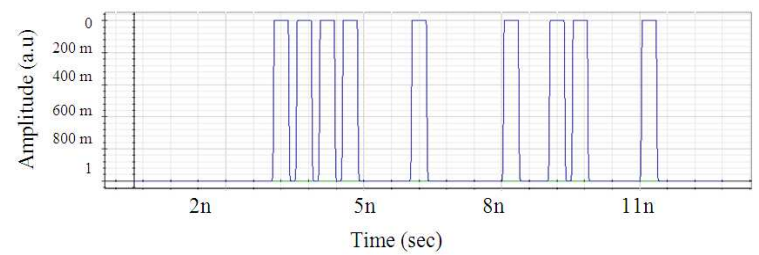

(a)

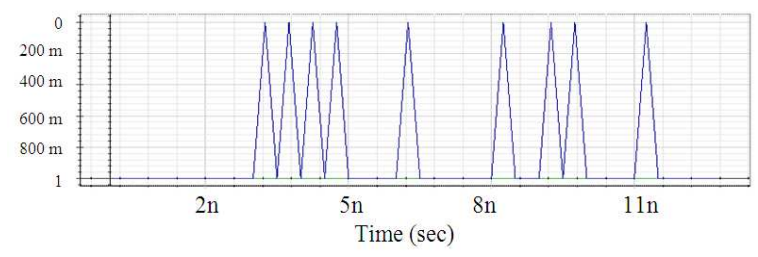

(b)

Fig. 1: Triangular and Rectangular (RZ) pulse modes

Input pulses and their correspondent coding: Electrical pulses induced by pulse generator, pulses then are sent to modulator (or diodes) to be converted into the optical signals. Here we represent three types of pulse shape that are used in our experiments:

- Rectangular pulse: It is considered as one of the most important and premier kinds of pulse form which is an idealization of real impulse signals mathematically. After modulation they are coded into optical pulses. The most common techniques for coding are NRZ (Non return to zero) and RZ (Return to zero)

- Triangular pulse: It is another important type of pulse forms. In some cases because it behaves more proper than rectangular form in some processes. One reason for this advantage is that its slopes are finite. The used coding in this experiment for triangular pulse shape is also RZ. In Fig. 1 we observe a sample of a similar input bits(...0111100100010110010...) and their correspondent rectangular and triangular coding

\section{MATERIALS AND METHODS}

The motivation of this study is to compare electrical dispersion compensation and optical one (with DCF). Also improvement of chromatic dispersion compensation techniques regarding to pulse formats will be investigated. For achieving this goal, we have simulated a point to point optical network with commercial simulator Optisystem 7.0 which is a product of the Optiwave Corporation. The utilized wavelength is $193.1 \mathrm{THz}$ that is produced by CW laser, which is modulated by a MZM modulator. External modulator is used because it is recommended for bitrate at $10 \mathrm{~Gb} \mathrm{sec}-1$ and higher. Via pulse generator, we apply NRZ, RZ and Triangular pulse form with data rate $10 \mathrm{~Gb} \mathrm{sec}^{-1}$. Injected power is considered $0 \mathrm{dBm}$. Table 1 represents other configurations.

It should be mentioned that common noises such as ASE and shot noise is applied to system be more actual. As we can observe, PMD has been disabled to just $\mathrm{CD}$ affects on the system. Also, since in single channel, higher order of GCD doesn't effect, they have been abandoned.

Performance parameters: In this study two performance parameters are used to assess the system performance: Quality Factor and Eye diagram. Q-factor is a dimensionless parameter which can be used to measure the quality of the signal. In Eq. 8, OOK system it correlates with BER mathematically with formula (Calvin and Chan, 2010):

$\mathrm{BER}=1 / 2 \operatorname{erfc}(\mathrm{Q} / \sqrt{2})$

Here erfc is error function. On the other hand eye diagram is a technique that provides an aggregation of signal measurement information and so helps us to evaluate the quality of the received signal. The quality of signal is assessed based on eye opening size. The vertical eye opening differentiates between signal level 0 and 1 . When this size is greater, it means the system can recognize signal levels 0 and 1 better. But if the vertical size of eye is too small, the system cannot differentiate 0 and 1 correctly. This problem occurs because of noise, dispersion and even attenuation.

The horizontal eye opening indicates the amount of jitter in the signal. Since jitter is an error in time domain, it can cause phase distortion and noise producing.

CD compensation with electrical equalizer: In this method we need to add an electrical compensator and low pass filter after photodetector. Also an electrical limiter is added exactly before our electrical compensator as can be seen in Fig. 2. In our experiment we utilized FFE-DFE method, because it is a simple method and consume power reasonably (Watts et al., 2005). In our experiment we consider Forward taps coefficients equal to 13 with taps space equal to 2 . Feedback taps coefficients were considered equal to 2 .

CD compensation with DCF: We can utilize a DCF and an extra amplifier for that. But since the length of the SSMF varies, we must vary the length of the DCF and its correspondent amplifier gain regarding to its length. Among the method on aspect of the place of DCF, we chose post-compensation. 


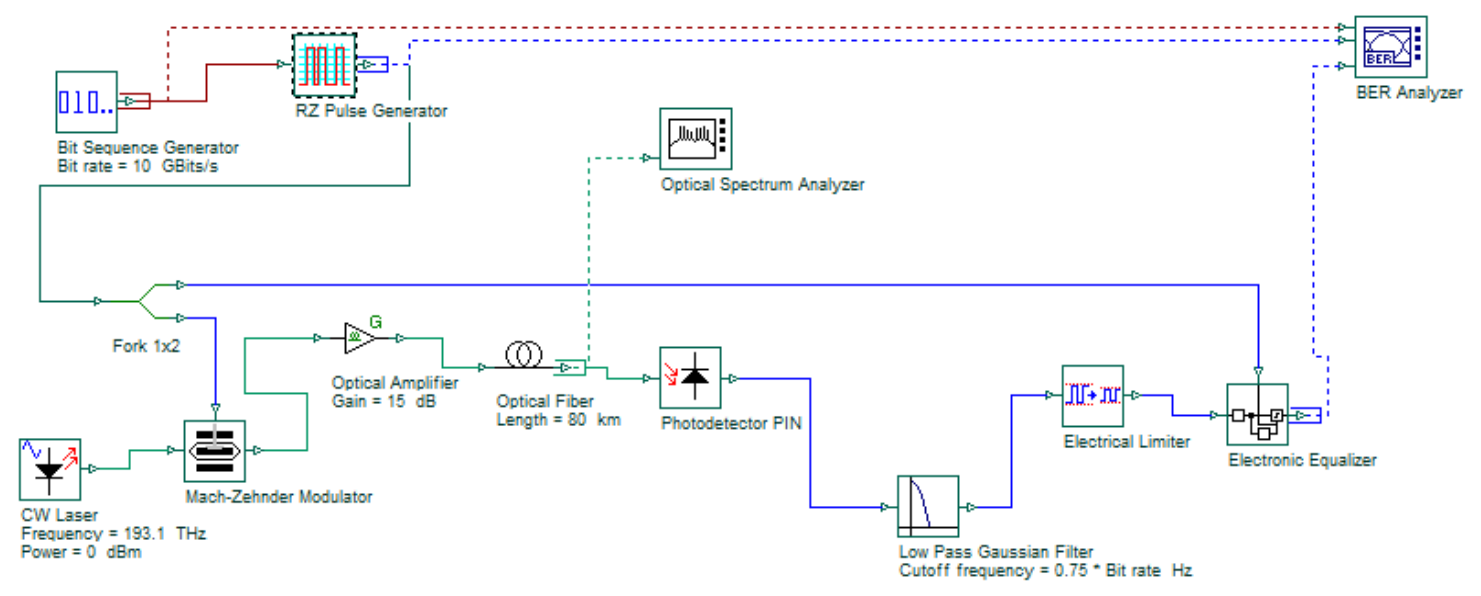

Fig. 2: A schema of CD compensation with electrical equalizer

Table 1: Summary of network components configurations

\begin{tabular}{|c|c|}
\hline Bit sequence generator & Bitrate: $10 \mathrm{~Gb} \mathrm{sec}{ }^{-1}$ \\
\hline Pulse Generator & (NRZ, RZ, Triangular) \\
\hline & Rectangular Shape: Gaussian -Duty Cycle: 0.67 bit \\
\hline $\mathrm{CW}$ laser & $\begin{array}{l}\text { Frequency: } 193.1 \mathrm{THz} \\
\text { - Power: } 0 \mathrm{dBm} \text {-Linewidth : } 10 \mathrm{MHz}\end{array}$ \\
\hline Mach-Zehnder modulator & Extinction Ratio: $10 \mathrm{~dB}$ \\
\hline Optical amplifier & Gain Control: $15 \mathrm{~dB}$ Noise Figure: $4 \mathrm{~dB}$ \\
\hline Optical fiber & Attenuation: $0.2 \mathrm{~dB} / \mathrm{km}$ GVD: $16 \mathrm{ps} / \mathrm{nm} \mathrm{km}$ PMD: 0 \\
\hline DC fiber & $\begin{array}{l}\text { Attenuation: } 0.6 \mathrm{~dB} / \mathrm{km}-\mathrm{GVD}:-90 \\
\mathrm{~s} / \mathrm{nm}-\mathrm{km} \text { - PMD: } 0\end{array}$ \\
\hline PIN photodetector & $\begin{array}{l}\text { Dark Current: } 10 \mathrm{nA} \\
\text {-Responsivity } 1 \mathrm{~A} / \mathrm{W}\end{array}$ \\
\hline Lowpass filter & $\begin{array}{l}\text { Cutoff Frequency: } \\
0.75 * \text { bitrate } \mathrm{HZ}\end{array}$ \\
\hline Electrical equalizer & $\begin{array}{l}\text { Reference bitrate } \\
10 \mathrm{~Gb} \mathrm{sec}^{-1} \text {-Step size: } 0.3\end{array}$ \\
\hline BER analyzer & Algorithm: gaussion- time window: 1.5 bit \\
\hline
\end{tabular}

Table 2: Required DCF length and correspondent amplifier

\begin{tabular}{lcr}
\hline $\begin{array}{l}\text { Length of } \\
\text { SMF }(\mathrm{km})\end{array}$ & $\begin{array}{l}\text { Required length } \\
\text { of DCF }(\mathrm{km})\end{array}$ & $\begin{array}{l}\text { Required amplifier } \\
\text { Gain }(\mathrm{dB})\end{array}$ \\
\hline 70 & 12.4 & 7.44 \\
80 & 14.2 & 8.52 \\
90 & 16.0 & 9.60 \\
100 & 17.7 & 10.62 \\
110 & 19.5 & 11.70 \\
120 & 21.3 & 12.78 \\
130 & 23.1 & 13.86 \\
140 & 24.8 & 14.88 \\
150 & 26.6 & 15.96 \\
160 & 28.4 & 17.04 \\
\hline
\end{tabular}

Because it has better performance compare to precompensation (Hu et al., 2010) (we don't have mixcompensation in one span that is implemented in our experiment).

Since the GVD in SSMF is $16 \mathrm{ps} \mathrm{km}^{-1} \mathrm{~nm}$ and in DCF is $-90 \mathrm{ps} \mathrm{km}^{-1} \mathrm{~nm}$ with formula 3.1 we can achieve the required length for DCF Eq. 9:

$\mathrm{L}_{\mathrm{DCF}}=\left(\mathrm{L}_{\mathrm{SMF}} \times \mathrm{D}_{\mathrm{SMF}}\right) / \mathrm{D}_{\mathrm{DCF}}$
On the other hand one of the most considerable properties of DCF is its high attenuation $\left(0.6 \mathrm{~dB} \mathrm{~km}^{-1}\right)$. of course for a fair result comparison between electrical and optical compensator, we need to add an amplifier to the link including of the DCF. Thus we determine the required extra gain of amplifier for a particular length of the optical link with following formula Eq. 10:

$\mathrm{G}=\alpha_{\mathrm{DCF}} \times \mathrm{L}_{\mathrm{DCF}}$

Since $\alpha \mathrm{DCF}=0.6 \mathrm{~dB} \mathrm{~km}^{-1}$ we can attain gain of amplifier for each length Eq. 11:

$\mathrm{G}=0.6 \times \mathrm{L}_{\mathrm{DCF}}$

Based on those formulas we provide a table (Table 2) that represent required length for DCF and also requires amplifier gain for that length.

\section{RESULTS}

CD compensation with electrical equalizer: From Fig. 3, we can observe from $70-160 \mathrm{~km}$ the network was implemented. With NRZ pulse shape, we observe the amount of Q-factor decreased with distance increment and after a little increment from 110-130 km we have a decrease again after $130 \mathrm{~km}$ so this situation continues until $160 \mathrm{~km}$ which signal Q-factor will be zero. It means the received signal has been fully distorted. If we consider the minimum acceptable Qfactor approximately 6 , we observe that with NRZ signal, maximum functional distance is $150 \mathrm{~km}$. If instead of NRZ, we utilize RZ pulse form (and consequently RZ coding), we will get rather similar performance. 
But if we use Triangular form of pulse we get a little different result. The maximum acceptable distance is fallen to $140 \mathrm{~km}$. As we can see we can attain the best performance with distance equal to $70 \mathrm{~km}$ for all three pulse modes.

\section{CD compensation with Optical Compensation} (DCF): If we consider value 6 for Q-factor limit as a good received signal, we observe in the graph as in Fig. 4, NRZ gives acceptable quality to the system and keeps the quality along the whole distance until $160 \mathrm{~km}$ which the signal quality crosses the minimum acceptable line and we cannot achieve this distance. RZ gives excellent quality to the system until $130 \mathrm{~km}$. With RZ received signal start to lose quality hugely. This state continues until $160 \mathrm{~km}$ that system will represent the minimum acceptable performance. Triangular pulse mode behaves similar RZ with this difference that the graph start to descend from almost $120 \mathrm{~km}$ and the system loses its acceptable quality after 160 . But along this process Triangular provides less signal quality compare to $\mathrm{RZ}$ and also we cannot achieve $160 \mathrm{~km}$ with minimum acceptable Q-factor.

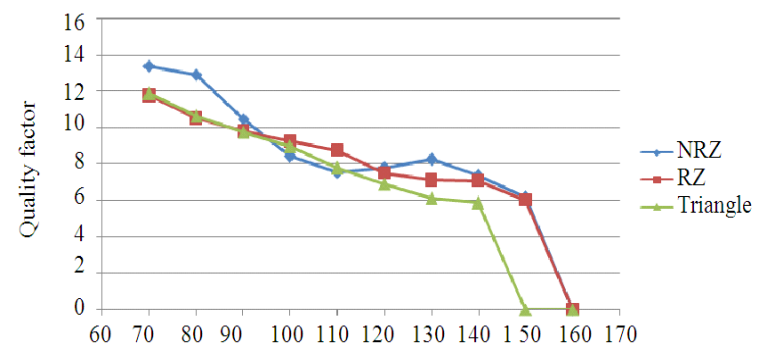

Fig. 3: CD Compensation with electrical equalizer

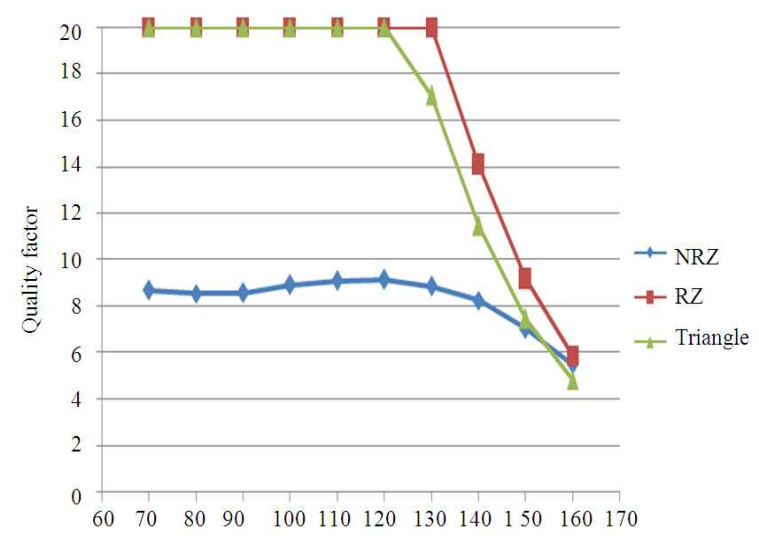

Fig. 4: CD compensation with variable DCF length

\section{DISCUSSION}

CD compensation with electrical equalizer: Overall we can say in electrical compensation, with shorter distance, we receive better quality of the signal. On aspect the different pulses, although RZ is more robustness against nonlinearities, we see from 70 until $90 \mathrm{~km}, \mathrm{NRZ}$ has better performance. It is because of this fact that NRZ is more robustness than $\mathrm{RZ}$ against chromatic dispersion (Forestieri, 2005). Except for 95-120 $\mathrm{km}$ that NRZ shows weakness against impairments, overall its strength predominates RZ; this result rather correspondents with Gene et al. (2007).

\section{CD compensation with Optical Compensation} (DCF): The reason of descend in RZ and Triangular from 130 until the end is because of the decrement of SNR at receiver.

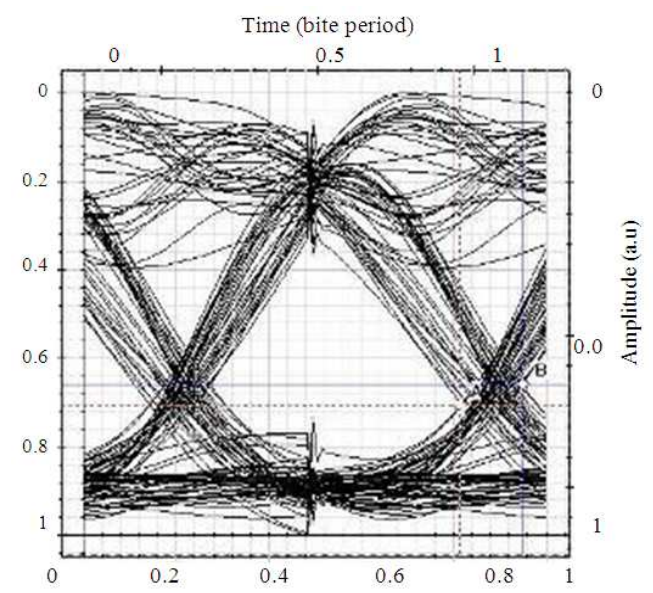

Fig. 5: Electrical compensation with NRZ, $70 \mathrm{~km}$ distance

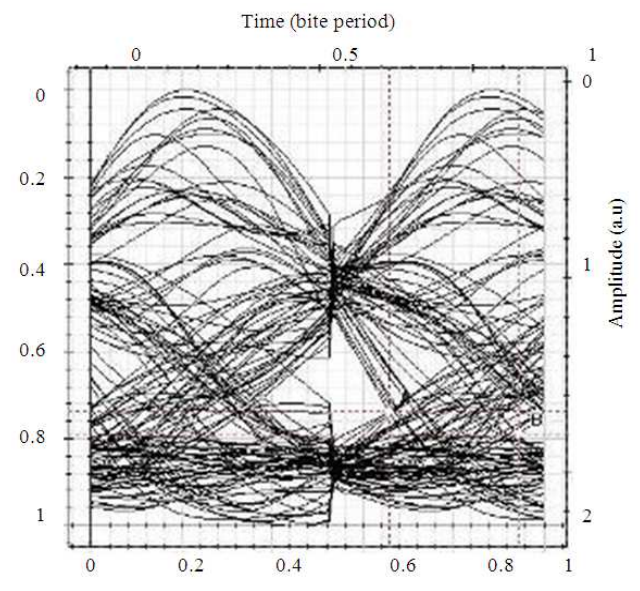

Fig. 6: Electrical compensation with NRZ, 140 km 


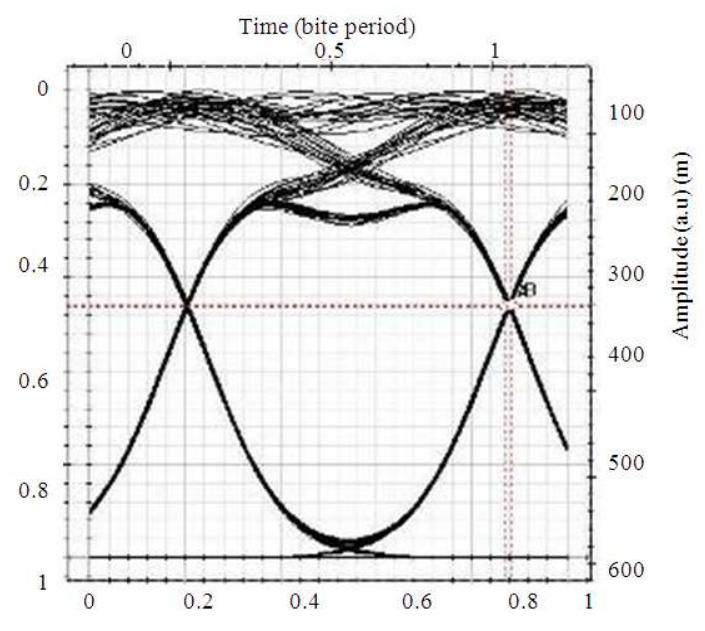

Fig. 7: Optical compensation with NRZ, $70 \mathrm{~km}$ distance

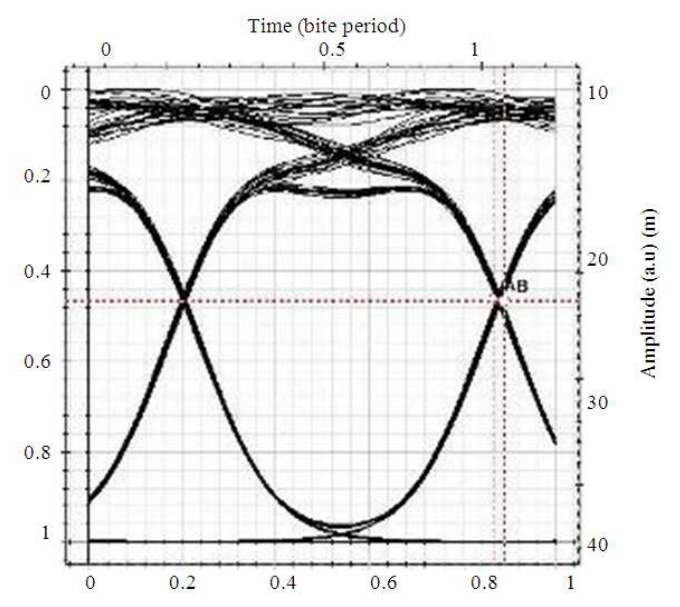

Fig. 8: optical compensation with NRZ, 140 km

Now, if we consider the distance from 70 to about 130 $\mathrm{km}$ where we have enough SNR, we can claim RZ is a little more resilient than Triangular form against combination of the nonlinear effects and noise and both are much better than NRZ (Breuer et al., 1996). On aspect of comparison between the performance of electrical equalizer and optical compensator with DCF, if our input pulse mode be NRZ, we observe that in distance of 70-90 km, electrical equalizer gives better response. But after that optical one excel than electrical. If our input be RZ or Triangular pulse form, in all situations optical compensator definitely is much better than electrical compensator and gives better performance. Overall based on our implementation; optical compensation superiors to electrical one.
Now we look on eye diagram for comparing between electrical and optical compensators in aspect of timing jitter. We want to observe how increment of distance affects timing jitter. Figure 7-8 are the eye diagram resulted from NRZ input pulse for electrical and optical compensation. As we observe in both Fig. 5 and 6, decision instant is implemented at 0.5 bit period.

So we scrutinize to timing jitter in right part of diagram (where electrical compensation has been applied). Observe after $70 \mathrm{~km}$ increment to distance, jitter has been surged considerably. The jitter was measured and while at $70 \mathrm{~km}$

jitter was 0.134532 portion of bit period, we get 0.28753 unit at $140 \mathrm{~km}$. it means the jitter has been increased 0.152998 portion of bit period. Now let's look at optical compensation with same situation; Figure 7-8. At $70 \mathrm{~km}$, we get jitter equal to 0.0131894 , and at $140 \mathrm{~km}$ we get 0.023741 portion of bit period.

It means jitter just increased 0.0105516 portion of bit period. This means even if increase the inserted power for SNR increment; we still cannot be hopeful for increment available distance with electrical compensator; because jitter will be a main obstacle, while it is not an issue for optical compensator. This result had been achieved by Pinto et al. (2006); (Breuer et al., 1996).

\section{CONCLUSION}

Optical networks are analogue in nature and suffer from a variety of linear and nonlinear transmission impairments. These effects have a direct impact on the Bit Error Rate (BER) performance of the system and most importantly, this impact increases in systems supporting higher data rates and larger number of channels (Papagiannakis et al., 2008).

In this study, chromatic dispersion compensation via electrical equalizer (with FFE-DFE method) and optical compensator (with DCF) and the role of electrical pulse mode in them was investigated. We concluded that in electrical compensation there is not big difference between inserted pulse mode, only we can say for shorter distance NRZ gives us good quality. But in optical, RZ and Triangular show better behavior and give us much higher performance than NRZ.

On aspect of electrical versus optical compensation, except for short distance with NRZ pulse mode, overall optical compensation gives us better performance. Also on aspect of jitter, electrical compensation shows that with distance increment, the jitter is accumulated which is a big problem for distance 
development in electrical compensations. As future work it is recommended to investigate the behavior and diversity of different pulses and modulations in negative and positive dispersion.

\section{ACKNOWLEDGEMENTS}

The researcher would like to acknowledge Universiti Kebangsaan Malaysia for supporting this research by providing equipment, material and sources of references in accomplishing this research and also also my supervisor Dr. Mohammad Syuhaimi AbRahman for his guides.

\section{REFERENCES}

Agrawal, G.P., 2002. Fiber-Optic Communications Systems. 3rd Edn., Wiley-Interscience, New York, ISBN: 0471215716, 546.

Bhandare, S., A. Joshi and D. Becker, 2010. Optical coherent receiver with a switchable electrical dispersion compensator for $10 \mathrm{~Gb} \sec ^{1}$ DPSK transmission up to $300 \mathrm{~km}$ of SSMF in metro optical networks. J. Lightwave Technol., 28: 47-58.

Breuer, D., K. Ennser and K. Petermann, 1996. Comparison of RZ- and RZ-modulation format for 40 Gbit $\sec ^{1}$ TDM standard-fibre systems. 22nd Eur. Conf. Optical Commun. ECOC, 2: 199-202.

Calvin, C. and Chan, 2010. Optical Performance Monitoring Advanced Techniques for NextGeneration Photonic Networks. 1st Edn. Academic Press/Elsevier, Burlington, ISBN: 9780123749505 pp: 482.

Forestieri, E., 2005. Optical communication theory and Techniques. 1st Edn. Springer, Italy, ISBN: 9780387231365 pp: 216

Gene, J.M., P.J. Winzer, S. Chandrasekhar and H. Kogelnik, 2007. Simultaneous compensation of polarization mode dispersion and chromatic dispersion using electronic signal processing. J. Lightwave Tech., 25: 1735-1741, DOI: 10.1109/JLT.2007.897751
Hu, B.N., W. Jing, W. Wei and R.M. Zhao, 2010. Analysis on dispersion compensation with DCF based on optisystem. Proceeding of the 2nd International Conference Industrial and Information Systems (IIS), 10-11 July, IEEE Xploor, Dalian, pp: 40-43, DOI: 10.1109/INDUSIS.2010.5565685

Keiser, G., 2003. Optical Communications Essentials. 1st Edn. McGraw-Hill, New York, ISBN: 0071412042, pp: 373.

Nuyts, R.J., Y.K. Park and P. Gallion, 1997. Dispersion equalization of a $10 \mathrm{~Gb} \mathrm{sec}^{1}$ repeatered transmission system using dispersion compensating fibers. J. Lightwave Tech., 15: 31-42. DOI: $10.1109 / 50.552111$

Papagiannakis, I., et al., 2008. Electronic distortion compensation in the mitigation of optical transmission impairments: the view of joint project on mitigation of optical transmission impairments by electronic means ePhoton/ONe1 project. IET Optoelectronics, 3: 73-85, DOI: 10.1049/ietopt.2008.0032

Pinto, A.N., J.R.F.D.A. Rocha, Q. Lin and G.P. Agrawal, 2006. Optical versus electrical dispersion compensation: role of timing jitter. J. Lightwave Tech., 24: 387.

Watts, P.M., V. Mikhailov, S. Savory, P. Bayvel and M. Glick, et al., 2005. Performance of single-mode fiber links using electronic feed-forward and decision feedback equalizers. IEEE Photon. Technol. Lett.,UK, 17: 2206-2208, DOI: 10.1109/LPT.2005.856326

Xu, T., G. Jacobsen, S. Popov, J. Li, K. Wang and A.T. Friberg, 2009. Digital Compensation of Chromatic Dispersion in 112-Gbit/s PDM-QPSK System. proceeding of the Asia Communications and Photonics Conference and Exhibition, Nov. OSA, Shanghai, China, pp: 1-3, 